\title{
Mitteilungen
}

\section{Hilfskasse für Schweizer Ärzte}

\section{Eingegangene Spenden}

Vom 1. April bis 30. Juni 2008 sind 28 Spenden im Gesamtbetrag von Fr. 23 791.- eingegangen. Der Stiftungsrat der Hilfskasse für Schweizer Ärzte freut sich sehr, diese Gaben bekanntgeben zu dürfen, und dankt allen Spenderinnen und Spendern recht herzlich. Damit die Spenden in voller Höhe den Destinatären zukommen, haben wir uns entschlossen, für Spenden unter Fr. 500.- auf den Versand von persönlichen Dankschreiben zu verzichten. Wir hoffen sehr, dass diese Massnahme bei allen Spendern auf Verständnis stösst.

Der Kassier des Stiftungsrates: Dr. med. Walter Zutter

\section{Caisse de secours}

\section{des médecins suisses}

\section{Contributions reçues}

Du $1^{\text {er }}$ avril au 30 juin 2008, nous avons reçu 28 dons d'une valeur totale de Fr. 23791.-. Le Conseil de fondation de la Caisse de secours des médecins suisses est très content de pouvoir publier ces chiffres et tient à remercier vivement tous les donateurs. Afin que les contributions puissent être versées entièrement aux destinataires, nous avons pris la décision de renoncer à l'envoi de lettres de remerciement pour les dons inférieurs à Fr. 500.-. Nous espérons que les donateurs feront preuve de compréhension et nous leur adressons encore une fois nos remerciements.

Le trésorier du Conseil de fondation: Dr Walter Zutter

\section{Société Vaudoise de Médecine} (SVM)

\section{Nouveau comité}

La composition complète du nouveau comité de la SVM est la suivante:

Président:

Dr Jean-Pierre Pavillon

(généraliste, Echallens)

Vice-président pour les libres praticiens:

Dr Charles A. Steinhäuslin

(chirurgien, Lausanne)

Vice-président pour les médecins hospitaliers:

Dr Lennart Magnusson

(anesthésiste, CHUV)

\section{Membres:}

Dr Hugues Burkhalter (généraliste, Lausanne); Dr Jean-Philippe Grob (interniste-hématologue, Lausanne); Dresse Véronique MonnierCornuz (gynécologue, Lausanne); Dr Bertrand Vuilleumier (chirurgien orthopédiste, Yverdon); Prof. Gérard Waeber (interniste-endocrinologue-diabétologue, professeur à la Faculté de biologie et de médecine, CHUV)

\section{Suchanfrage}

Deutscher Medizinhistoriker, der an einer Buchreihe über die Schweizer Ärztemissionen im 2. Weltkrieg schreibt, sucht noch nach Dokumenten und Fotos bei ehemaligen Teilnehmern. Er bittet um Mithilfe bei der Suche nach den Angehörigen folgender Schweizer Kollegen: 1. Prof. André Calame, Genf, verst. 1999 in Chancy; 2. Dr. Max Arnold, verst. 1956, seit 1936 Chefarzt der Heilstätte «Du Midi» in Davos; 3. Dr. André Lenoir, verst. 1978, aus Genf, 1942 Radiologe am Kantonsspital Zürich; 4. Dr. Eduard Jäger, 1941 am Kantonspital Glarus, wohnte in Zürich, Sonnenbergstrasse 55 . Wer kannte die Kollegen und kann Hinweise zu den Angehörigen geben? Nachricht bitte an Dr. med. Reinhold Busch, Ruhreckstraße 13, D-58099 Hagen, Tel. +492331 6890 92, Fax +49 23319699 81, E-Mail: dr.rbusch@gmx.de

\section{Hermann-Emminghaus-Preis 2009}

Medizinerinnen und Mediziner, die wissenschaftliche Arbeiten auf dem Gebiet der Kinder- und Jugendpsychiatrie und -psychotherapie verfasst haben, können sich ab sofort für den Hermann-Emminghaus-Preis 2009, der seit 1995 von der Lilly Deutschland GmbH, Bad Homburg, gesponsert wird, bewerben bzw. dafür vorgeschlagen werden. Die alle zwei Jahre verliehene Auszeichnung würdigt seit knapp einem Vierteljahrhundert hervorragende wissenschaftliche Leistungen des Fachgebiets. Der Preisträger erhält die Hermann-Emminghaus-Medaille und ein mit 5500 Euro dotiertes Preisgeld.

Teilnahmeberechtigt sind Ärztinnen und Ärzte, die grundlegende wissenschaftliche Arbeiten in deutscher Sprache auf dem Gebiet der Diagnostik, der Prognostik oder der Therapie psychischer Erkrankungen im Kindes- und Jugendalter verfasst haben. Psychodynamisch, genetisch oder zerebralorganisch orientierte Untersuchungen kommen ebenso in Betracht wie epidemiologische, katamnestische oder therapeutische Studien.

Die Auswahl der Preisträger erfolgt durch das Kuratorium des Hermann-Emminghaus-Preises bestehend aus vier Vertretern des Fachgebiets und zwei von Lilly Deutschland benannten Mitgliedern. Die Auszeichnung wird zum Gedenken an Hermann Emminghaus, den Pionier der kinder- und jugendpsychiatrischen Forschung, im Rahmen der 31. Jahrestagung der Deutschen Gesellschaft für Kinderund Jugendpsychiatrie, Psychosomatik und Psychotherapie (DGKJP) Anfang März 2009 in Hamburg vergeben.

Bewerbungen in sechsfacher Ausfertigung können bis spätestens 1. November 2008 unter folgender Adresse eingereicht werden: Kuratorium Hermann-Emminghaus-Preis, c/o Fuhrmann \& Schütz Healthcare, Public Relations GmbH \& Co. KG, Herr Steffen Fritzsche, Humboldtstraße 11, D-65189 Wiesbaden, Tel. +49611 16086 40, Fax +49611 16086 15, E-Mail: fritzsche@fuhrmannschuetz.de.

Weitere Informationen zum Hermann-Emminghaus-Preis finden Sie im Internet unter www.emminghaus-preis.de. 